\title{
Article
}

\section{On Bilinear Narrow Operators}

\author{
Marat Pliev ${ }^{1, * \mathbb{D}}$, Nonna Dzhusoeva ${ }^{2}$ and Ruslan Kulaev ${ }^{1,2} \mathbb{D}$ \\ 1 Southern Mathematical Institute, Russian Academy of Sciences, 362027 Vladikavkaz, Russia; \\ kulaev@smath.ru \\ 2 Department of Mathematics and Computer Sciences, North-Ossetian State University Named after K.L. \\ Khetagurov, 362025 Vladikavkaz, Russia; djusoevanonna@rambler.ru \\ * Correspondence: plimarat@yandex.ru
}

Citation: Pliev, M.; Dzhusoeva, N.; Kulaev, R. On Bilinear Narrow Operators. Mathematics 2021, 9, 2892. https://doi.org/10.3390/ math9222892

Academic Editor: Juan Benigno Seoane-Sepúlveda

Received: 12 October 2021 Accepted: 10 November 2021 Published: 13 November 2021

Publisher's Note: MDPI stays neutral with regard to jurisdictional claims in published maps and institutional affiliations.

Copyright: (c) 2020 by the authors. Licensee MDPI, Basel, Switzerland. This article is an open access article distributed under the terms and conditions of the Creative Commons Attribution (CC BY) license (https:/ / creativecommons.org/licenses/by/ $4.0 /)$.

\begin{abstract}
In this article, we introduce a new class of operators on the Cartesian product of vector lattices. We say that a bilinear operator $T: E \times F \rightarrow W$ defined on the Cartesian product of vector lattices $E$ and $F$ and taking values in a vector lattice $W$ is narrow if the partial operators $T_{x}$ and $T_{y}$ are narrow for all $x \in E, y \in F$. We prove that, for order-continuous Köthe-Banach spaces $E$ and $F$ and a Banach space $X$, the classes of narrow and weakly function narrow bilinear operators from $E \times F$ to $X$ are coincident. Then, we prove that every order-to-norm continuous $C$-compact bilinear regular operator $T$ is narrow. Finally, we show that a regular bilinear operator $T$ from the Cartesian product $E \times F$ of vector lattices $E$ and $F$ with the principal projection property to an order continuous Banach lattice $G$ is narrow if and only if $|T|$ is.
\end{abstract}

Keywords: bilinear operator; narrow operator; order-to-norm continuous operator; $C$-compact operator; regular operator; Köthe-Banach space; vector lattice

\section{Introduction}

Linear narrow operators on function spaces can be considered as a generalization of compact operators. The foundation of the theory of these operators is presented in [1]. The aim of this article is to extend the concept of narrowness to the setting of bilinear operators.

Here, we provide some necessary facts and notations which we need in our further presentation. For all unexplained notions and notations, we refer the reader to [2,3]. All the vector lattices we consider below are supposed to be Archimedean.

Two elements $x$ and $y$ of a vector lattice $W$ are said to be disjoint (notation $x \perp y$ ) if $|x| \wedge|y|=0$. We write $x=\bigsqcup_{i=1}^{n} x_{i}$, if $x=\sum_{i=1}^{n} x_{i}$ and $x_{i} \perp x_{j}$ for all $i \neq j$. In particular, if $n=2$, we use the notation $x=x_{1} \sqcup x_{2}$. We say that $y$ is a fragment (a component) of $x \in E$ and use the notation $y \sqsubseteq x$, if $y \perp(x-y)$. The set of all fragments of an element $x \in E$ we denote by $\mathfrak{F}_{x}$. We say that $x_{1}, x_{2} \in \mathfrak{F}_{x}$ are mutually complemented, if $x=x_{1} \sqcup x_{2}$.

A measure space $(A, \Sigma, \mu)$ is said to be finite if $\mu(A)<\infty$. Let $(A, \Sigma, \mu)$ be a finite measure space. A Banach space $E$, which is an order ideal in $L_{0}(A, \Sigma, \mu)$ with a lattice norm, is said to be a Köthe-Banach space on $(A, \Sigma, \mu)$. A Köthe-Banach space $E$ over $(A, \Sigma, \mu)$ is said to have order continuous norm if $\lim _{n \rightarrow \infty}\left\|e_{n}\right\|=0$ for any sequence $\left(e_{n}\right)_{n \in \mathbb{N}} \subset E$, such that $e_{n} \downarrow 0$. For a given $f \in L_{0}(\mu)$ by $\operatorname{supp} f$, we denote the measurable set

$$
\operatorname{supp} f:=\{t \in A: f(t) \neq 0\} .
$$

The characteristic function of a set $D$ is denoted by $1_{D}$. The union $H \cup D$ of two disjoint sets $H$ and $D$ we denote by $H \sqcup D$. The vector space of all bounded bilinear operators from the Cartesian product $E \times F$ of normed spaces $E$ and $F$ to a Banach space $W$ we denote by $\mathcal{B}(E, F ; W)$. The closed unit ball of a Banach space $X$ is denoted by $B_{X}$. The vector space of all linear bounded operators on $X$ is denoted by $\mathcal{L}(X)$. 
Definition 1. Let $E, F$ and $W$ be vector spaces. With an operator $T: E \times F \rightarrow W$ are associated two families of partial operators $T_{x}: F \rightarrow W, x \in E$ and $T_{y}: E \rightarrow W, y \in F$ defined by

$$
T_{x}(v):=T(x, v), \quad v \in F ; \quad T_{y}(u):=T(u, y), u \in E .
$$

We say that $T: E \times F \rightarrow W$ is a bilinear operator if all $T_{x}: F \rightarrow W, x \in E$ and $T_{y}: E \rightarrow W$, $y \in F$ are linear operators from $E$ to $W$ and from $F$ to $W$, respectively.

Given vector lattices $E$ and $F$ by $E \times F$ is denoted the Cartesian product $E \times F:=\{(x, y)$ : $x \in E, y \in F\}$, that is, a vector lattice with the pointwise algebraic and lattice operations. Precisely, for all $x, u \in E$ and $y, v \in F$, we have that

$$
\begin{array}{r}
(x, y) \leq(u, v) \Leftrightarrow x \leq u \text { and } y \leq v ; \\
(x, y) \vee(u, v)=(x \vee u, y \vee v) ;(x, y) \wedge(u, v)=(x \wedge u, y \wedge v) ; \\
|(x, y)|=(|x|,|y|) .
\end{array}
$$

Let us suppose that $E, F$ and $W$ are vector lattices. We say that a bilinear operator $T: E \times F \rightarrow W$ is: 1. Positive, if $T\left(E_{+} \times F_{+}\right) \in W_{+}$;

2. Regular, if $T=S_{1}-S_{2}$, where $S_{1}$ and $S_{2}$ are positive bilinear operators from $E \times F$ to $W$. The vector space (cone) of all regular (positive) bilinear operators from $E \times F$ to $W$ we denote by $B_{r}(E, F ; W)(B+(E, F ; W))$.

We note that a bilinear operator $T: E \times F \rightarrow W$ does not need to be linear as an operator defined on a vector lattice $E \times F$. Indeed, let us suppose that $(x, y)$ and $(u, v)$ are some elements of $E \times F$. Then, we may write

$$
\begin{gathered}
T((x, y)+(u, v))=T(x+u, y+v)=T(x, y)+ \\
T(x, v)+T(u, y)+T(u, v) \neq T(x, y)+T(u, v) .
\end{gathered}
$$

Let us suppose that $E, F$ and $W$ are vector lattices. We note that a regular bilinear operator $T: E \times F \rightarrow W$ can be viewed as a regular linear operator from a vector lattice $E$ to a vector lattice $L_{r}(F, W)$ of all regular linear operators from $F$ to $W$. If a vector lattice $W$ is Dedekind complete, then, by the Riesz-Kantorovich theorem ([3] (Theorem 1.18)), we have that $B_{r}(E, F ; W)$ is a Dedekind complete vector lattice.

\section{Bilinear Narrow Operators on Köthe-Banach Spaces}

In this section, we consider narrow, function narrow and function weakly narrow bilinear operators defined on the Cartesian products of Köthe-Banach spaces and taking values in a Banach space.

Definition 2. Let $(A, \Sigma, \mu)$ be a finite measure space, E be a Köthe-Banach space on $(A, \Sigma, \mu)$ and $X$ be a normed space. A bounded linear operator $S: E \rightarrow X$ is called:

1. Function narrow, if, for every $D \in \Sigma$ and $\varepsilon>0$, there exists a disjoint decomposition $D=D_{1} \sqcup D_{2}$ with $D_{1}, D_{2} \in \Sigma$ such that $\mu\left(D_{1}\right)=\mu\left(D_{2}\right)$ and $\left\|S\left(1_{D_{1}}-1_{D_{2}}\right)\right\|_{X}<\varepsilon$;

2. Function weakly narrow, if, for every $D \in \Sigma$ and $\varepsilon>0$, there exists a disjoint decomposition $D=D_{1} \sqcup D_{2}$ with $D_{1}, D_{2} \in \Sigma$ such that $\left\|S\left(1_{D_{1}}-1_{D_{2}}\right)\right\|_{X}<\varepsilon$;

3. Narrow, if, for every $f \in E$ and $\varepsilon>0$, there exist mutually complemented fragments $f_{1}, f_{2} \in \mathcal{F}_{f}$ such that $\left\|S\left(f_{1}-f_{2}\right)\right\|_{X}<\varepsilon$.

Definition 3. Let finite measure spaces $E$ and $F$ be Köthe-Banach spaces on $(A, \Sigma, \mu)$ and $(B, \Theta, v)$, respectively, and $X$ be a normed space. A bounded bilinear $T: E \times F \rightarrow X$ is called function weakly narrow (function narrow, narrow), if, for every $u \in E, v \in F$ partial operators $T_{v}$ and $T_{u}$ are. 
For Banach spaces $E, F$ and $X$ by $\|T\|_{E \times F \rightarrow X}$, we denote the norm of a bounded bilinear $T: E \times F \rightarrow X$. That is,

$$
\|T\|_{E \times F \rightarrow X}:=\sup \left\{\|T(e, f)\|_{X}:\|e\|_{E} \leq 1,\|f\|_{F} \leq 1\right\} .
$$

Clearly,

$$
\|T(e, f)\|_{X} \leq\|T\|_{E \times F \rightarrow X}\|e\|_{E}\|f\|_{F} \text { for all } e \in E, f \in F .
$$

We observe that the notion of a narrow operator on a Köthe-Banach space can be extended to the setting of operators defined on vector lattices.

Definition 4. Let $E$ be a vector lattice and $X$ be a normed space. A linear operator $S: E \rightarrow X$ is called narrow, if, for every $x \in E$ and $\varepsilon>0$, there exist mutually complemented fragments $x_{1}$ and $x_{2}$ of $x$, such that $\left\|S\left(x_{1}-x_{2}\right)\right\|<\varepsilon$.

Since every element $x \in E$ has the representation $x=x_{+}-x_{-}$, we note that an element $x$ in Definition 4 can be taken from $E_{+}$. Indeed, let us suppose $x_{+}^{1}, x_{+}^{2}$ and $x_{-}^{1}, x_{-}^{2}$ are two pairs of mutually complemented fragments of $x_{+}$and $x_{-}$, respectively, such that $\left\|S\left(x_{+}^{1}-x_{+}^{2}\right)\right\|<\frac{\varepsilon}{2}$ and $\left\|S\left(x_{-}^{1}-x_{-}^{2}\right)\right\|<\frac{\varepsilon}{2}$. Let us put $x_{1}=x_{+}^{1}-x_{-}^{1}$ and $x_{2}=x_{+}^{2}-x_{-}^{2}$. It is clear that $x_{1}$ and $x_{2}$ are mutually disjoint fragments of $x$. Now, we have that

$$
\begin{gathered}
\left\|S\left(x_{1}-x_{2}\right)\right\|=\left\|S\left(x_{+}^{1}-x_{-}^{1}-x_{+}^{2}+x_{-}^{2}\right)\right\| \leq \\
\left\|S\left(x_{+}^{1}-x_{+}^{2}\right)\right\|+\left\|S\left(x_{-}^{1}-x_{-}^{2}\right)\right\|<\frac{\varepsilon}{2}+\frac{\varepsilon}{2}=\varepsilon .
\end{gathered}
$$

We note that a narrow operator $S$ maps atoms of $E$ to zero of $X$. Indeed, since, for every atom $x \in E_{+}$, there exists a unique disjoint decomposition $x=x \sqcup 0$ of $x$. It follows that $\|S x\|<\varepsilon$ for every $\varepsilon>0$; therefore, $S x=0$. Due to these circumstances, the study of narrow operators is of interest only on atomless vector lattices.

Definition 5. Let $E$ and $F$ be vector lattices, $X$ be a normed space and $T: E \times F \rightarrow X$ be a bilinear operator. We say that an operator $T$ is narrow, if, for all $u \in E, v \in F$ partial operators, $T_{v}$ and $T_{u}$ are narrow.

The next theorem is the main result of this section.

Theorem 1. Let $(A, \Sigma, \mu)$ and $(B, \Xi, v)$ be finite atomless measure spaces, $E$ and $F$ be KötheBanach spaces on $(A, \Sigma, \mu)$ and $(B, \Xi, v)$ equipped with order continuous norms $\|\cdot\|_{E}$ and $\| \cdot$ $\|_{F}$, respectively, $X$ be a Banach space and $T \in \mathcal{B}(E, F ; X)$. Then, the following statements are equivalent:

1. $T$ is a narrow operator;

2. $T$ is a function weakly narrow operator.

Proof. $(2) \Rightarrow(1)$. Let us pick $g \in F$. We prove the narrowness of $T_{g}: E \rightarrow X, g \in F$. Fix $f \in E_{+}$and $\varepsilon>0$. We need to find two mutually complemented fragments $f_{1}$ and $f_{2}$ of $f$ such that $\left\|T_{g}\left(f_{1}-f_{2}\right)\right\|_{X}<\varepsilon$. We note that there exists a sequence simple functions $\xi_{n}=\sum_{k=1}^{k(n)} r_{k}^{n} 1_{A_{k}^{n}}$, such that $0 \leq \xi_{n} \uparrow f, n \in \mathbb{N}$. By the order continuity of the norm $\|\cdot\|_{E}$, there exists $n_{0} \in \mathbb{N}$ and $\xi_{n_{0}}=\sum_{k=1}^{k\left(n_{0}\right)} r_{k}^{n_{0}} 1_{A_{k}^{n_{0}}}$, such that $\left\|f-\xi_{n_{0}}\right\|_{E}<$ $\frac{\varepsilon}{3\|T\|_{E \times F \rightarrow X}\|g\|_{F}}$ for every $n \in \mathbb{N}, n \geq n_{0}$. By the weakly function narrowness of $T_{g}$, there exists a disjoint decomposition: $A_{k}^{n_{0}}=\Delta_{k}^{n_{0}} \sqcup \Theta_{k}^{n_{0}}, \Delta_{k}^{n_{0}}, \Theta_{k}^{n_{0}} \in \Sigma$, where $1 \leq k \leq k\left(n_{0}\right)$, such that $\left\|T_{g}\left(r_{k}^{n_{0}} 1_{\Delta_{k}^{n_{0}}}-r_{k}^{n_{0}} 1_{\Theta_{k}^{n_{0}}}\right)\right\|<\frac{\varepsilon}{3 k\left(n_{0}\right)}$. Let us put 


$$
\begin{array}{r}
u_{n_{0}}=\sum_{k=1}^{k\left(n_{0}\right)} r_{k}^{n_{0}} 1_{\Delta_{k}^{n_{0}}} ; v_{n_{0}}=\sum_{k=1}^{k\left(n_{0}\right)} r_{k}^{n_{0}} 1_{\Theta_{k}^{n_{0}}} ; \\
\Delta=\bigsqcup_{k=1}^{k\left(n_{0}\right)} \Delta_{k}^{n_{0}} ; \Theta=\operatorname{supp} f \backslash((\operatorname{supp} f) \cap \Delta)
\end{array}
$$

Let us suppose that $u=f 1_{\Delta}$ and $v=f 1_{\Theta}$. It is clear that $u$ and $v$ are mutually complemented fragments of $f$. Since $\left(u-u_{n_{0}}\right) \perp\left(v-v_{n_{0}}\right)$, we have that

$$
\begin{aligned}
& f-\xi_{n_{0}}=\left(u-u_{n_{0}}\right) \sqcup\left(v-v_{n_{0}}\right) \geq u-u_{n_{0}} ; \\
& f-\xi_{n_{0}}=\left(u-u_{n_{0}}\right) \sqcup\left(v-v_{n_{0}}\right) \geq v-v_{n_{0}} .
\end{aligned}
$$

Since $\|\cdot\|_{E}$ is a lattice norm, we have that $\left\|f-\xi_{n_{0}}\right\|_{E} \geq\left\|u-u_{n_{0}}\right\|_{E}$ and $\left\|f-\xi_{n_{0}}\right\|_{E} \geq$ $\left\|v-v_{n_{0}}\right\|_{E}$. Now, we may write

$$
\begin{array}{r}
\left\|T_{g}(u-v)\right\|_{X}= \\
\left\|T_{g} u-T_{g} v-T_{g} u_{n_{0}}+T_{g} u_{n_{0}}+T_{g} v_{n_{0}}-T_{g} v_{n_{0}}\right\|_{X} \leq \\
\left\|T_{g}\left(u-u_{n_{0}}\right)\right\|_{X}+\left\|T_{g}\left(v-v_{n_{0}}\right)\right\|_{X}+\left\|T_{g}\left(u_{n_{0}}-v_{n_{0}}\right)\right\|_{X} \leq \\
\|T\|_{E \times F \rightarrow X}\left\|u-u_{n_{0}}\right\|_{E}\|g\|_{F}+\|T\|_{E \times F \rightarrow X}\left\|v-v_{n_{0}}\right\|_{E}\|g\|_{F}+ \\
\left\|\sum_{k=1}^{k\left(n_{0}\right)} T_{g}\left(x_{k}^{n_{0}} 1_{\Delta_{k}^{n_{0}}}-x_{k}^{n_{0}} 1_{\left.\Theta_{k}^{n_{0}}\right)}\right)\right\|_{X} \leq \\
\|T\|_{E \times F \rightarrow X}\left\|f-\xi_{n_{0}}\right\|_{E}\|g\|_{F}+\|T\|_{E \times F \rightarrow X}\left\|f-\xi_{n_{0}}\right\|_{E}\|g\|_{F}+ \\
\sum_{k=1}^{k\left(n_{0}\right)}\left\|T_{g}\left(x_{k}^{n_{0}} 1_{\Delta_{k}^{n_{0}}}-x_{k}^{n_{0}} 1_{\left.\Theta_{k}^{n_{0}}\right)}\right)\right\|_{X}< \\
\frac{\varepsilon}{3}+\frac{\varepsilon}{3}+\frac{\varepsilon}{3}=\varepsilon .
\end{array}
$$

Putting $f_{1}=u$ and $f_{2}=v$, we have established the narrowness of $T_{g}$. Similarly, for every $f \in E$ it can be proved the narrowness of $T_{f}: F \rightarrow X$.

The implication $(1) \Rightarrow(2)$ is obvious.

We note that the problem of coincidence of classes of function narrow and function weakly operators still remains open, even for the linear case (see [4]).

\section{C-Compact and Narrow Operators}

In this section, we consider $C$-compact bilinear operators and show that $C$-compactness of a bilinear operator implies its narrowness. We note that $C$-compact operators on vector lattices and lattice-normed spaces were investigated recently in [5-7].

Definition 6. Let $E$ be a vector lattice, $X$ be a normed space and $T: E \rightarrow X$ be a linear operator. We say that $T$ is:

1. $C$-compact, if, for every $x \in E$, an operator $T$ maps $\mathfrak{F}_{x}$ to a relatively compact set in $F$;

2. Order-to-norm continuous, if $T$ maps every order convergent net $\left(x_{\alpha}\right)_{\alpha \in \Lambda}$ in $E$, with $x=\lim _{\alpha} x_{\alpha}$, to a norm convergent net $\left(T x_{\alpha}\right)_{\alpha \in \Lambda}$ in $F$, which converges to $T x$.

Definition 7. Let $E$ and $F$ be vector lattices and $X$ be a normed space. A bilinear operator $T: E \times F \rightarrow X$ is called C-compact (order-to-norm continuous), if, for all $u \in E, v \in F$ partial operators $T_{u}: F \rightarrow X, T_{v}: E \rightarrow X$ are C-compact (order-to-norm continuous).

The following theorem is the main result of this section. 
Theorem 2. Let $E$ and $F$ be atomless Dedekind complete vector lattices, $X$ be a Banach space and $T: E \times F \rightarrow X$ be an order-to-norm continuous $C$-compact bilinear operator. Then, $T$ is a narrow operator.

For the proof of Theorem 2, we need some auxiliary propositions.

Proposition 1. ([1] (Lemma 10.20)) Let $\left(v_{i}\right)_{i=1}^{n}$ be a family of elements of a finite-dimensional vector space $Y$ and let $\left(\lambda_{i}\right)_{i=1}^{n}$ be a family of real numbers such that $0 \leq \lambda_{i} \leq 1$ for every $i$. Then, there exists a family of reals $\left(\theta_{i}\right)_{i=1}^{n}$, with $\theta_{i} \in\{0,1\}$, such that

$$
\left\|\sum_{i=1}^{n}\left(\lambda_{i}-\theta_{i}\right) v_{i}\right\| \leq \frac{\operatorname{dim} Y}{2} \max _{i}\left\|v_{i}\right\| .
$$

Let us suppose that $E$ is a Dedekind complete vector lattice $E$ and $x \in E_{+}$. Then, $\mathfrak{F}_{x}$ is an order-complete Boolean algebra equipped with the natural order induced from $E$ ([3] (Theorem 1.49)).

Proposition 2. Let $E$ and $F$ be atomless Dedekind complete vector lattices, $x \in E_{+}, v \in F$ $\left(y \in F_{+}, u \in E\right), X$ be a Banach space and $T: E \times F \rightarrow X$ be an order-to-norm continuous bilinear operator. Then, for every $\varepsilon>0$, there exists a disjoint decomposition $x=z \sqcup h(y=w \sqcup p)$ such that $\left\|T_{v} z\right\|<\varepsilon\left(\left\|T_{u} w\right\|<\varepsilon\right)$.

Proof. We prove the assertion for $T_{v}: E \rightarrow X, v \in F$. Since $E$ is atomless, we have that a Boolean algebra $\mathfrak{F}_{x}$ has an infinite cardinality. The net of fragments $\left(x_{\alpha}\right)_{\alpha \in \Lambda}$, where $\Lambda=\mathfrak{F}_{x}$ order converges to $x$. By the order-to-norm continuity of $T_{v}$, there exists a nonzero fragment $x_{\alpha_{0}}$ such that $\left\|T_{v}\left(x-x_{\alpha}\right)\right\|<\varepsilon$ for every $\alpha \geq \alpha_{0}$. Let us put $z=x_{\alpha_{0}}$ and $h=x-x_{\alpha_{0}}$. The similar proof is valid for the partial operator $T_{u}: F \rightarrow X$.

Proposition 3. Let $E$ and $F$ be atomless Dedekind complete vector lattices, $x \in E_{+}, v \in F\left(y \in F_{+}\right.$, $u \in E), \varepsilon>0, X$ be a Banach space and $T: E \times F \rightarrow X$ be an order-to-norm continuous bilinear operator. Then, we can choose $n \in \mathbb{N}(m \in \mathbb{N})$ such that there exists a disjoint decomposition $x=\bigsqcup_{i=1}^{n} x_{i}\left(y=\bigsqcup_{j=1}^{m} y_{j}\right)$, where $x_{i}\left(y_{j}\right)$ are fragments of $x(y)$ such that $\left\|T_{v} x_{i}\right\| \leq \varepsilon\left(\left\|T_{u} y_{j}\right\| \leq \varepsilon\right)$ for every $i \in\{1, \ldots, n\}(j \in\{1, \ldots, m\})$.

Proof. We prove the assertion for a partial operator $T_{v}: E \rightarrow X, v \in F$. If $\left\|T_{v} x\right\| \leq \varepsilon$, then we may assume that $n=1$; there is nothing to prove. Let us assume that $\left\|T_{v} x\right\|>\varepsilon$. By Proposition 2, the set

$$
D_{x, T_{v}, \varepsilon}:=\left\{z \in \mathfrak{F}_{x}: z \neq 0,\left\|T_{v} z\right\| \leq \varepsilon\right\}
$$

is non-empty. We note that $D_{x, T_{v}, \varepsilon}$ is equipped with a partial order $\leq$, induced from $\mathfrak{F}_{x}$. Let us suppose that $\left(u_{\gamma}\right)_{\gamma \in \Gamma} \subseteq D_{x, T_{v}, \varepsilon}$ is a chain where $\Gamma$ is an index set. Taking into account the order completeness of a Boolean algebra $\mathfrak{F}_{x}$ and the order-to-norm continuity of $T_{v}$, we have that $u^{\star}=\sup _{\gamma}\left(u_{\gamma}\right) \in \mathfrak{F}_{x}$ and $\left\|T_{v} u^{\star}\right\| \leq \varepsilon$. Hence, $u^{\star} \in D_{x, T_{v}, \varepsilon}$. By the Zorn lemma, there exists a maximal element $x^{D} \in D_{x, T_{v}, \varepsilon}$. If $\left\|T_{v}\left(x-x^{D}\right)\right\| \leq \varepsilon$, then, putting $x_{0}:=x$ and $x_{0}^{D}:=x^{D}$, we have the desired disjoint decomposition $x=x_{0}=x_{0}^{D} \sqcup\left(x_{0}-x_{0}^{D}\right)$. Otherwise, applying Proposition 2 to the element $x_{1}:=x_{0}-x_{0}^{D}$, we can find a decomposition $x_{1}=x_{1}^{D} \sqcup x_{2}$, where $x_{1}^{D}$ is the maximal element of $D_{x_{1}, T_{v}, \varepsilon}$. Proceeding further, we construct a sequence of subsets $A_{k}, k \in \mathbb{N}$, where

$$
\begin{array}{r}
A_{k}:=\left\{x_{0}^{D}, \ldots, x_{k}^{D}, x_{k+1}\right\} \text { with } x=\bigsqcup_{i=0}^{k} x_{i}^{D} \sqcup x_{k+1} \\
\text { and }\left\|T_{v}\left(x_{i}^{D}\right)\right\| \leq \varepsilon \text { for all } i \in\{0, \ldots, k\} .
\end{array}
$$


We claim that there exists $l \in \mathbb{N}$ such that $\left\|T_{v}\left(x_{l+1}\right)\right\| \leq \varepsilon$. First, we show that the sequence $\left(x_{k}\right)_{k \in \mathbb{N}}$ order converges to 0 . Indeed, assuming the contrary, we can find $0<\xi \leq x_{k}$ for all $k \in \mathbb{N}$. Then, there is a sequence

$$
x_{1}^{\prime}:=x_{1}-\xi, \ldots, x_{k}^{\prime}:=x_{k}-\xi, \ldots
$$

such that $x_{n}^{\prime} \sqsubseteq x_{m}^{\prime}, n \geq m ; m, n \in \mathbb{N}$ and $\inf _{k \in \mathbb{N}} x_{k}^{\prime}=\{0\}$. By the order-to-norm continuity of $T_{v}$, there exists $n_{0} \in \mathbb{N}$ such that $\left\|T_{v}\left(x_{n_{0}}^{\prime}\right)\right\|<\varepsilon$. Hence, $x_{n_{0}}=x_{n_{0}}^{\prime} \sqcup \xi$ and $x_{n_{0}}^{\prime} \in D_{\zeta_{n_{0}}, T_{v}, \varepsilon}$. Since $x_{n_{0}}^{D}$ is the maximal element of $D_{x_{n_{0}}, T_{v}, \varepsilon}$, the inequality $x_{n_{0}}^{D}<x_{n_{0}}^{\prime}$ is impossible and either $x_{n_{0}}^{\prime}=x_{n_{0}}^{D}$ or the set $\mathfrak{F}_{x_{n_{0}}^{D}} \cap \mathfrak{F}_{\xi}$ contains a non-zero fragment. In the first case, $x_{n_{0}+1}=\xi$; therefore, $x_{n_{0}+2}$ is a fragment of $\xi$ and $0<x_{n_{0}+2}<\xi$. In the second case, $\xi$ is not a fragment of $x_{n_{0}+1}$. Hence, we come to a contradiction; consequently, $\inf _{k \in \mathbb{N}} x_{k}=\{0\}$. Then, by the order-to-norm continuity of $T_{v}$, we have that $\lim _{k \rightarrow \infty}\left\|T_{v}\left(x_{k}\right)\right\|=0$. It follows that there exists $l \in \mathbb{N}$ such that $\left\|T_{v}\left(x_{l}\right)\right\| \leq \varepsilon$. Thus,

$$
\begin{array}{r}
A_{l}:=\left\{x_{0}^{D}, \ldots, x_{l}^{D}, x_{l+1}\right\} \text { with } x=\bigsqcup_{i=0}^{l} x_{i}^{D} \sqcup x_{l+1}, \\
\left\|T_{v}\left(x_{i}^{D}\right)\right\| \leq \varepsilon \text { for all } i \in\{0, \ldots, k\}, \text { and }\left\|T_{v}\left(x_{l+1}\right)\right\| \leq \varepsilon
\end{array}
$$

is the required disjoint decomposition of $x$. The existence of a disjoint decomposition $y=\bigsqcup_{j=1}^{m} y_{j}$ such that $\left\|T_{u} y_{j}\right\| \leq \varepsilon$ for every $j \in\{1, \ldots, m\}$ can be proved in the same way as above.

We observe that, by arbitrariness of $\varepsilon>0$ in the inequalities $\left\|T_{v} x_{i}\right\| \leq \varepsilon, i \in\{1, \ldots, n\}$ $\left(\left\|T_{u} y_{j}\right\| \leq \varepsilon, j \in\{1, \ldots, m\}\right)$, we may assume that $\left\|T_{v} x_{i}\right\|<\varepsilon, i \in\{1, \ldots, n\}\left(\left\|T_{u} y_{j}\right\|<\varepsilon\right.$, $j \in\{1, \ldots, m\})$.

Proposition 4. Let $E$ and $F$ be atomless Dedekind complete vector lattices and $Y$ be a finitedimensional Banach space. Then, every order-to-norm continuous bilinear operator $T: E \times F \rightarrow Y$ is narrow.

Proof. Let us fix $v \in F$ and show the narrowness of $T_{v}: E \rightarrow Y$. Let us pick $x \in E_{+}$ and $\varepsilon>0$. By Proposition 3, there exists a disjoint decomposition $x=\bigsqcup_{i=1}^{n} x_{i}$ such that $\left\|T_{v} x_{i}\right\|<\frac{\varepsilon}{\operatorname{dim} Y}$ for every $1 \leq i \leq n$. Then, by Proposition 1 , we can find $\lambda_{i} \in\{0,1\}$, $1 \leq i \leq n$, such that

$$
\left\|\sum_{1 \leq i \leq n}\left(1-2 \lambda_{i}\right) T_{v} x_{i}\right\|=2\left\|\sum_{i=1}^{n}\left(\frac{1}{2}-\lambda_{i}\right) T_{v} x_{i}\right\| \leq \operatorname{dim} Y \max _{1 \leq \mathrm{i} \leq \mathrm{n}}\left\|\mathrm{T}_{\mathrm{v}} \mathrm{x}_{\mathrm{i}}\right\| .
$$

Let $J_{1}=\left\{1 \leq i \leq n: \lambda_{i}=0\right\}$ and $J_{2}=\left\{1 \leq i \leq n: \lambda_{i}=1\right\}$. Then, $z_{j}=\sum_{i \in J_{j}} x_{i}, j=1,2$, are mutually complemented fragments of $x$ and, by (1), we have that

$$
\begin{array}{r}
\left\|T_{v}\left(z_{1}-z_{2}\right)\right\|=\left\|T_{v}\left(\sum_{i \in J_{1}} x_{i}-\sum_{i \in J_{2}} x_{i}\right)\right\|= \\
\left\|\sum_{1 \leq i \leq n}\left(1-2 \lambda_{i}\right) T_{v} x_{i}\right\| \leq \operatorname{dim} Y \max _{1 \leq i \leq n}\left\|T_{v} x_{i}\right\|<\varepsilon .
\end{array}
$$

Hence, the narrowness of $T_{v}$ is proved. The narrowness of $T_{u}, u \in E$ can be proved analogously.

Definition 8. Let $E$ and $F$ be vector lattices and $X$ be a vector space. We say that $T: E \times F \rightarrow X$ is a bilinear operator of a finite rank, if the subspace of $X$ generated by $T(E \times F)$ is finite-dimensional. 
Now, we are ready to prove the main result of this section.

Proof of Theorem 2. As above, we prove the narrowness of a partial operator $T_{v}: E \rightarrow X$, $v \in F$. We recall that a Banach space $X$ can be considered as a closed vector subspace of a Banach space $l_{\infty}\left(B_{X^{\star}}\right)$ of the space all bounded functions on a compact topological space $B_{X^{\star}}$. Then, there is isometric embedding of $X$ in $W:=l_{\infty}\left(B_{X^{\star}}\right)$. Let us suppose that $D$ is a set of the infinite cardinality. We observe that, for a relatively compact subset $H$ of $l_{\infty}(D)$ and $\varepsilon>0$, there exists a linear operator of a finite rank $S \in \mathcal{L}\left(l_{\infty}(D)\right)$, such that $\|x-S x\| \leq \varepsilon$ for every $x \in H$ ([1] (Lemma 10.25)). Let us pick $x \in E_{+}$and $\varepsilon>0$. Since $T_{v}$ is a $C$-compact linear operator, it follows that $K:=T_{v}\left(\mathfrak{F}_{x}\right)$ is the relatively compact subset in $X$; therefore, $l_{\infty}\left(B_{X^{\star}}\right)$. Then, there exists a linear bounded operator of a finite rank $S \in \mathcal{L}\left(l_{\infty}\left(B_{X^{*}}\right)\right)$ such that $\|w-S w\| \leq \frac{\varepsilon}{4}$ for every $w \in K$. Then, $R=S \circ T$ is an order-to-norm continuous bilinear operator of a finite rank and $R_{v}=S \circ T_{v}$ is the partial operator of $R$, that acts from $E$ to $X$. By Proposition 4, there exist mutually complemented fragments $x_{1}, x_{2}$ of $x$ such that $\left\|R_{v} x_{1}-R_{v} x_{2}\right\|<\frac{\varepsilon}{2}$. Then, we may write

$$
\begin{aligned}
&\left\|T_{v} x_{1}-T_{v} x_{2}\right\|= \\
&\left\|T_{v} x_{1}-T_{v} x_{2}+S\left(T_{v} x_{1}\right)-S\left(T_{v} x_{2}\right)-S\left(T_{v} x_{1}\right)+S\left(T_{v} x_{2}\right)\right\|= \\
&\left\|T_{v} x_{1}-T_{v} x_{2}+R_{v} x_{1}-R_{v} x_{2}-S\left(T_{v} x_{1}\right)+S\left(T_{v} x_{2}\right)\right\| \leq \\
&\left\|R_{v} x_{1}-R_{v} x_{2}\right\|+\left\|T_{v} x_{1}-S\left(T_{v} x_{1}\right)\right\|+\left\|T_{v} x_{2}-S\left(T_{v} x_{2}\right)\right\|< \\
& \frac{\varepsilon}{2}+\frac{\varepsilon}{4}+\frac{\varepsilon}{4}=\varepsilon .
\end{aligned}
$$

Hence, the narrowness of $T_{v}$ is proved. The narrowness of a partial operator $T_{u}: F \rightarrow X$, $u \in E$ can be proved analogously.

\section{A Regular Bilinear Operator $T$ Is Narrow If and Only If $|T|$ Is a Narrow Operator}

In this section, we investigate the relationship between the narrowness of a regular bilinear operator $T: E \times F \rightarrow G$ and the narrowness of its modulus $|T|: E \times F \rightarrow G$.

The next theorem is the main result of this section.

Theorem 3. Let $E$ and $F$ be vector lattices with the principal projection property, $G$ be a Banach lattice with an order continuous norm and $T: E \times F \rightarrow G$ be a regular bilinear operator. Then, the following statements are equivalent:

1. T is a narrow operator;

2. $|T|$ is a narrow operator.

For the proof, we need the following auxiliary propositions.

Proposition 5. ([8] (Prop. 2.5.,2.7)) Let $E$ and $F$ be vector lattices with the principal projection property, $G$ be a Dedekind complete vector lattice, $x \in E_{+}, y \in F_{+}$and $T \in B_{r}(E, F ; G)$. Then, the following equality holds:

$$
|T|(x, y)=\sup \left\{\sum_{i=1}^{n} \sum_{j=1}^{m}\left|T\left(x_{i}, y_{j}\right)\right|: x=\bigsqcup_{i=1}^{n} x_{i} ; y=\bigsqcup_{j=1}^{m} y_{j} ; m, n \in \mathbb{N}\right\} .
$$

We note that $\left\{\sum_{i=1}^{n} \sum_{j=1}^{m}\left|T\left(x_{i}, y_{j}\right)\right|: x=\bigsqcup_{i=1}^{n} x_{i} ; y=\bigsqcup_{j=1}^{m} y_{j} ; m, n \in \mathbb{N}\right\}$ is the upwarddirected set for every $x \in E_{+}$and $y \in F_{+}$.

Proposition 6. Let $(A, \Sigma, \mu)$ be a finite measure space, J be an order ideal of $L_{1}(\mu)$ with an order continuous norm $\|\cdot\|_{J}$ and $\left(w_{n}\right)_{n \in \mathbb{N}}$ be a sequence in $J$. If a sequence $\left\|w_{n}\right\|_{L_{1}(\mu)}$ converges to 0 , then there exists a subsequence $\left(w_{n_{k}}\right)_{k \in \mathbb{N}}$ of $\left(w_{n}\right)_{n \in \mathbb{N}}$ such that $\left\|w_{n_{k}}\right\|_{J}$ converges to 0 . 
Proof. Since $\left\|w_{n}\right\|_{L_{1}(\mu)} \rightarrow 0$, it follows that a sequence $\left(w_{n}\right)_{n \in \mathbb{N}}$ converges by measure to 0 ; therefore, there exists a subsequence $\left(w_{n_{k}}\right)_{k \in \mathbb{N}}$ of $\left(w_{n}\right)_{n \in \mathbb{N}}$ such that $\left(w_{n_{k}}\right)_{k \in \mathbb{N}}$ order converges to 0 . By the order continuity of the norm $\|\cdot\|_{J}$, we obtain $\left\|w_{n_{k}}\right\|_{J} \rightarrow 0$.

Proof of Theorem 3. (1) $\Rightarrow(2)$. Let us fix $y \in F_{+}$. We need to show that the partial operator $|T(\cdot, y)|: E \rightarrow G$ is narrow. Let us fix an element $x \in E_{+}$and $\varepsilon>0$. Since the set

$$
\left\{\sum_{i=1}^{n} \sum_{j=1}^{m}\left|T\left(x_{i}, y_{j}\right)\right|: x=\bigsqcup_{i=1}^{n} x_{i} ; y=\bigsqcup_{j=1}^{m} y_{j} ; n, m \in \mathbb{N}\right\}
$$

is upward-directed and the norm of $F$ is order-continuous by Proposition 5, there exist, $n \in \mathbb{N}, m \in \mathbb{N}$, disjoint decompositions $x=\bigsqcup_{i=1}^{n} x_{i}$ and $y=\bigsqcup_{j=1}^{m} y_{j}$ such that

$$
\left\||T|(x, y)-\sum_{i=1}^{n} \sum_{j=1}^{m}\left|T\left(x_{i}, y_{j}\right)\right|\right\|<\frac{\varepsilon}{3}
$$

By the narrowness of $T$, we see that partial operators $T\left(\cdot, y_{j}\right)$ are narrow for every $j \in\{1, \ldots, m\}$; therefore, for each $i \in\{1, \ldots, n\}$, there exists a disjoint decomposition $x_{i}=x_{i}^{1} \sqcup x_{i}^{2}$ such that $\left\|T\left(x_{i}^{1}, y_{j}\right)-T\left(x_{i}^{2}, y_{j}\right)\right\|<\frac{\varepsilon}{3(n+m)}$. Let us assign, by definition,

$$
w=\bigsqcup_{i=1}^{n} x_{i}^{1} \text { and } h=\bigsqcup_{i=1}^{n} x_{i}^{2} .
$$

Clearly, $w$ and $h$ are mutually complemented fragments of $x$. Taking into account that

$$
\begin{array}{r}
|T|(x, y)=|T|(w, y)+|T|(h, y) \\
|T|(w, y) \geq \sum_{i=1}^{n} \sum_{j=1}^{m}\left|T\left(x_{i}^{1}, y_{j}\right)\right| \geq 0 \\
\text { and }|T|(h, y)-\sum_{i=1}^{n} \sum_{j=1}^{m}\left|T\left(x_{i}^{2}, y_{j}\right)\right| \geq 0
\end{array}
$$

we have that

$$
\begin{array}{r}
0 \leq|T|(x, y)\left|-\sum_{i=1}^{n} \sum_{j=1}^{m}\right| T\left(x_{i}^{1}, y_{j}\right)\left|-\sum_{i=1}^{n} \sum_{j=1}^{m}\right| T\left(x_{i}^{2}, y_{j}\right) \mid \leq \\
|T|(x, y)-\sum_{i=1}^{n} \sum_{j=1}^{m}\left|T\left(x_{i}, y_{j}\right)\right| \Rightarrow\left\||T|(w, y)-\sum_{i=1}^{n} \sum_{j=1}^{m}\left|T\left(x_{i}^{1}, y_{j}\right)\right|\right\| \leq \\
\left\||T|(x, y)-\sum_{i=1}^{n} \sum_{j=1}^{m}\left|T\left(x_{i}, y_{j}\right)\right|\right\|<\frac{\varepsilon}{3} \text { and } \\
\left\||T|(h, y)-\sum_{i=1}^{n} \sum_{j=1}^{m}\left|T\left(x_{i}^{2}, y_{j}\right)\right|\right\| \leq\left\||T|(x, y)-\sum_{i=1}^{n} \sum_{j=1}^{m}\left|T\left(x_{i}, y_{j}\right)\right|\right\|<\frac{\varepsilon}{3} .
\end{array}
$$


Now, we may write

$$
\begin{array}{r}
\||T|(w, y)-|T|(h, y)\|= \\
\||T|(w, y)-\sum_{i=1}^{n} \sum_{j=1}^{m}\left|T\left(x_{i}^{1}, y_{j}\right)\right|+\sum_{i=1}^{n} \sum_{j=1}^{m}\left|T\left(x_{i}^{1}, y_{j}\right)\right|- \\
|T|(h, y)+\sum_{i=1}^{n} \sum_{j=1}^{m}\left|T\left(x_{i}^{2}, y_{j}\right)\right|-\sum_{i=1}^{n} \sum_{j=1}^{m}\left|T\left(x_{i}^{2}, y_{j}\right)\right| \| \leq \\
\left\||T|(w, y)-\sum_{i=1}^{n} \sum_{j=1}^{m}\left|T\left(x_{i}^{1}, y_{j}\right)\right|\right\|+\left\||T|(h, y)+\sum_{i=1}^{n} \sum_{j=1}^{m}\left|T\left(x_{i}^{2}, y_{j}\right)\right|\right\|+ \\
\left\|\sum_{i=1}^{n} \sum_{j=1}^{m}\left|T\left(x_{i}^{1}, y_{j}\right)\right|-\sum_{i=1}^{n} \sum_{j=1}^{m}\left|T\left(x_{i}^{2}, y_{j}\right)\right|\right\| \leq \\
\frac{\varepsilon}{3}+\frac{\varepsilon}{3}+\sum_{i=1}^{n} \sum_{j=1}^{m}\left\|T\left(x_{i}^{1}, y\right)-T\left(x_{i}^{2}\right), y\right\|<\varepsilon .
\end{array}
$$

Hence, $x=w \sqcup h$ is the required disjoint decomposition of $x$ and $|T(\cdot, y)|$ is a narrow operator. The narrowness of $|T(x, \cdot)|$ can be proved in the same way as above for every $x \in E$.

(2) $\Rightarrow$ (1). Let us fix again $x \in E_{+}, y \in F_{+}$and $\varepsilon>0$. If $|T(x, y)|=0$, then $T(x, y)=0$; there is nothing to prove. Thus, we may assume that $|T|(x, y)>0$. Let us consider a sequence $\left(\varepsilon_{k}\right)_{k \in \mathbb{N}}$ in $\mathbb{R}_{+}$with $\varepsilon_{k} \downarrow 0$. By Proposition 5 and the order continuity of $\|\cdot\|_{G}$, there exist $n(k) \in \mathbb{N}, m(k) \in \mathbb{N}$ and a pair of sequences of disjoint decompositions $x=\bigsqcup_{i=1}^{n(k)} x_{i}^{k}$ and $y=\bigsqcup_{j=1}^{m(k)} y_{j}^{k}$ of $x$ and $y$, respectively, such that

$$
\left\||T|(x, y)-\sum_{i=1}^{n(k)} \sum_{j=1}^{m(k)}\left|T\left(x_{i}^{k}, y_{j}^{k}\right)\right|\right\|<\frac{\varepsilon_{k}}{4} .
$$

By the narrowness of $|T|$, for each $\left(x_{i}^{k}, y_{j}^{k}\right)$, where $i \in\{1, \ldots, n(k)\}$ and $j \in\{1, \ldots, m(k)\}$, there exists a disjoint decomposition $x_{i}^{k}=x_{i}^{1 k} \sqcup x_{i}^{2 k}$ such that $\left\||T|\left(x_{i}^{1 k}, y_{j}^{k}\right)-|T|\left(x_{i}^{2 k}, y_{j}^{k}\right)\right\|<$ $\frac{\varepsilon_{k}}{4(n(k)+m(k))}$. Let us assign, by definition, $u_{k}=\bigsqcup_{i=1}^{n(k)} x_{i}^{1 k}$ and $v_{k}=\bigsqcup_{i=1}^{n(k)} x_{i}^{2 k}$. We observe that, for every $k \in \mathbb{N}$, we have that

$$
\begin{array}{r}
0 \leq|T|\left(u_{k}, y\right)-\sum_{i=1}^{n(k)} \sum_{j=1}^{m(k)}\left|T\left(x_{i}^{1 k}, y_{j}^{k}\right)\right| \leq \\
|T|(x, y)-\sum_{i=1}^{n(k)} \sum_{j=1}^{m(k)}\left(\left|T\left(x_{i}^{1 k}, y_{j}^{k}\right)\right|+\left|T\left(x_{i}^{2 k}, y_{j}^{k}\right)\right|\right) \leq \\
|T|(x, y)-\sum_{i=1}^{n(k)} \sum_{j=1}^{m(k)}\left|T\left(x_{i}^{k}, y_{j}^{k}\right)\right| .
\end{array}
$$

Similar arguments show that

$$
0 \leq|T|\left(v_{k}, y\right)-\sum_{i=1}^{n(k)} \sum_{j=1}^{m(k)}\left|T\left(x_{i}^{2 k}, y_{j}^{k}\right)\right| \leq|T|(x, y)-\sum_{i=1}^{n(k)} \sum_{j=1}^{m(k)}\left|T\left(x_{i}^{k}, y_{j}^{k}\right)\right| .
$$

We observe that $|T(w, z)| \leq|T|(x, y)$ for all $w \in \mathfrak{F}_{x}$ and $z \in \mathfrak{F}_{y}$. By $\pi_{x, y}$, we denote the order projection onto the band generated by $|T|(x, y)$. Then, $I=\pi_{x, y} G$ is a Banach lattice with 
an order continuous norm equipped with the weak order unit $|T|(x, y)$. By ([9] (Theorem 1.b.14)), there exist a probability space $(\Omega, \Sigma, \mu)$ and an order ideal $J$ of $L_{1}(\mu)$ such that $I$ is lattice-isometric to $J$. Thus, it is enough to prove the inequality $\|T(\psi, y)-T(\phi, y)\|_{J}<\varepsilon$ for some $\psi, \phi$, where $x=\psi \sqcup \phi$. We note that, for every $u, v \in L_{1}(\mu)$, the following equality holds:

$$
\|u-v\|_{L_{1}(\mu)}=\||u|-|v|\|_{L_{1}(\mu)}+\|u\|_{L_{1}(\mu)}+\|v\|_{L_{1}(\mu)}-\|u+v\|_{L_{1}(\mu)} .
$$

Now, we may write

$$
\begin{array}{r}
\left\|T\left(u_{k}, y\right)-T\left(v_{k}, y\right)\right\|_{L_{1}(\mu)}= \\
\left\|T\left(\bigsqcup_{i=1}^{n(k)} x_{i}^{1 k}, \bigsqcup_{j=1}^{m(k)} y_{j}^{k}\right)-T\left(\bigsqcup_{i=1}^{n(k)} x_{i}^{2 k}, \bigsqcup_{j=1}^{m(k)} y_{j}^{k}\right)\right\|_{L_{1}(\mu)}= \\
\left\|\sum_{i=1}^{n(k)} \sum_{j=1}^{m(k)} T\left(x_{i}^{1 k}, y_{j}^{k}\right)-\sum_{i=1}^{n(k)} \sum_{j=1}^{m(k)} T\left(x_{i}^{2 k}, y_{j}^{k}\right)\right\|_{L_{1}(\mu)} \leq \\
\sum_{i=1}^{n(k)} \sum_{j=1}^{m(k)}\left\|T\left(x_{i}^{1 k}, y_{j}^{k}\right)-T\left(x_{i}^{2 k}, y_{j}^{k}\right)\right\|_{L_{1}(\mu)} .
\end{array}
$$

We observe that the $L_{1}(\mu)$-norm of a sum of positive elements is equal to the sum of their norms. Now, applying Equation (2) to the last formula and taking into account that $\|u\|=\||u|\|$ for every $u \in L_{1}(\mu)$, we obtain

$$
\begin{array}{r}
\sum_{i=1}^{n(k)} \sum_{j=1}^{m(k)}\left\|T\left(x_{i}^{1 k}, y_{j}^{k}\right)-T\left(x_{i}^{2 k}, y_{j}^{k}\right)\right\|_{L_{1}(\mu)}= \\
\sum_{i=1}^{n(k)} \sum_{j=1}^{m(k)}\left\|\left|T\left(x_{i}^{1 k}, y_{j}^{k}\right)\right|-\left|T\left(x_{i}^{2 k}, y_{j}^{k}\right)\right|\right\|_{L_{1}(\mu)}+\sum_{i=1}^{n(k)} \sum_{j=1}^{m(k)}\left\|T\left(x_{i}^{1 k}, y_{j}^{k}\right)\right\|_{L_{1}(\mu)}+ \\
\sum_{i=1}^{n(k)} \sum_{j=1}^{m(k)}\left\|T\left(x_{i}^{2 k}, y_{j}^{k}\right)\right\|_{L_{1}(\mu)}-\sum_{i=1}^{n(k)} \sum_{j=1}^{m(k)}\left\|T\left(x_{i}^{1 k}, y_{j}^{k}\right)+T\left(x_{i}^{2 k}, y_{j}^{k}\right)\right\|_{L_{1}(\mu)} .
\end{array}
$$

It follows that

$$
\begin{array}{r}
\sum_{i=1}^{n(k)} \sum_{j=1}^{m(k)}\left\|T\left(x_{i}^{1 k}, y_{j}^{k}\right)-T\left(x_{i}^{2 k}, y_{j}^{k}\right)\right\|_{L_{1}(\mu)}= \\
\sum_{i=1}^{n(k)} \sum_{j=1}^{m(k)} \|\left|T\left(x_{i}^{1 k}, y_{j}^{k}\right)\right|-|T|\left(x_{i}^{1 k}, y_{j}^{k}\right)+|T|\left(x_{i}^{1 k}, y_{j}^{k}\right)- \\
|T|\left(x_{i}^{2 k}, y_{j}^{k}\right)+|T|\left(x_{i}^{2 k}, y_{j}^{k}\right)-\left|T\left(x_{i}^{2 k}, y_{j}^{k}\right)\right| \|_{L_{1}(\mu)}+ \\
\sum_{i=1}^{n(k)} \sum_{j=1}^{m(k)}\left\|\left|T\left(x_{i}^{1 k}, y_{j}^{k}\right)\right|\right\|_{L_{1}(\mu)}+\sum_{i=1}^{n(k)} \sum_{j=1}^{m(k)}\left\|\left|T\left(x_{i}^{2 k}, y_{j}^{k}\right)\right|\right\|_{L_{1}(\mu)}- \\
\sum_{i=1}^{n(k)} \sum_{j=1}^{m(k)}\left\|\left|T\left(x_{i}^{k}, y_{j}^{k}\right)\right|\right\|_{L_{1}(\mu)} .
\end{array}
$$


Then, we have the following estimates:

$$
\begin{array}{r}
\sum_{i=1}^{n(k)} \sum_{j=1}^{m(k)}\left\|T\left(x_{i}^{1 k}, y_{j}^{k}\right)-T\left(x_{i}^{2 k}, y_{j}^{k}\right)\right\|_{L_{1}(\mu)} \leq \sum_{i=1}^{n(k)} \sum_{j=1}^{m(k)}\left\||T|\left(x_{i}^{1 k}, y_{j}^{k}\right)-\left|T\left(x_{i}^{1 k}, y_{j}^{k}\right)\right|\right\|_{L_{1}(\mu)}+ \\
\sum_{i=1}^{n(k)} \sum_{j=1}^{m(k)}\left\||T|\left(x_{i}^{2 k}, y_{j}^{k}\right)-\left|T\left(x_{i}^{2 k}, y_{j}^{k}\right)\right|\right\|_{L_{1}(\mu)}+\sum_{i=1}^{n(k)} \sum_{j=1}^{m(k)} \||T|\left(x_{i}^{1 k}, y_{j}^{k}\right)- \\
|T|\left(x_{i}^{2 k}, y_{j}^{k}\right)\left\|_{L_{1}(\mu)}+\sum_{i=1}^{n(k)} \sum_{j=1}^{m(k)}\right\||T|\left(x_{i}^{1 k}, y_{j}^{k}\right) \|_{L_{1}(\mu)}+ \\
\left\||T|\left(u_{k}, y\right)-\sum_{i=1}^{n(k)}\left|T\left(x_{i}^{1 k}, y\right)\right|\right\|_{L_{1}(\mu)}+\left\||T|\left(v_{k}, y\right)-\sum_{i=1}^{n(k)}\left|T x_{i}^{2 k}, y\right|\right\|_{L_{1}(\mu)}+\frac{\varepsilon_{k}}{4}+ \\
\sum_{i=1}^{n(k)} \sum_{j=1}^{m(k)}\left\||T|\left(x_{i}^{1 k}, y_{j}^{k}\right)+|T|\left(x_{i}^{2 k}, y_{j}^{k}\right)\right\|_{L_{1}(\mu)}-\sum_{i=1}^{n(k)} \sum_{j=1}^{m(k)}\left\|\left|T\left(x_{i}^{k}, y_{j}^{k}\right)\right|\right\|_{L_{1}(\mu)}< \\
\frac{\varepsilon_{k}}{4}+\frac{\varepsilon_{k}}{4}+\frac{\varepsilon_{k}}{4}+\left\||T|(x, y)-\sum_{i=1}^{n(k)} \sum_{j=1}^{m(k)}\left|T\left(x_{i}^{k}, y_{j}^{k}\right)\right|\right\|_{L_{1}(\mu)}<\varepsilon_{k} .
\end{array}
$$

Thus, for every $k \in \mathbb{N}$, we have that $\left\|T\left(u_{k}, y\right)-T\left(v_{k}, y\right)\right\|_{L_{1}(u)}<\varepsilon_{k}$. Let us put $w_{n}=T\left(u_{k}, y\right)-T\left(v_{k}, y\right), k \in \mathbb{N}$. Then, the sequence $\left(\left\|w_{k}\right\|_{L_{1}(\mu)}\right)$ converges to 0 and, by Proposition 6 , there exists a subsequence $\left(w_{k_{l}}\right)_{l \in \mathbb{N}}$ such that $\left\|w_{k_{l}}\right\|_{J}$ converges to 0 . Hence, there exists $l_{0} \in \mathbb{N}$ such that $\left\|T\left(u_{k_{l}}, y\right)-T\left(v_{k_{l}}, y\right)\right\|_{J}<\varepsilon$ for all $l \geq l_{0}$. Let us put $r=k_{l_{0}}$. Then,

$$
\left\|T\left(u_{r}, y\right)-T\left(v_{r}, y\right)\right\|_{J}=\left\|T\left(u_{k_{l_{0}}}, y\right)-T\left(v_{k_{l_{0}}}, y\right)\right\|_{J}<\varepsilon
$$

and $x=u_{r} \sqcup v_{r}$ is the required disjoint decomposition of $x$ into the sum of two mutually complemented fragments.

\section{Concluding Remarks}

Linear narrow operators were first introduced and studied by Plichko and Popov in [10]. However, these operators were previously explored by different authors (Kalton, Rosenthal and others); see the monograph [1] and references therein. It was shown in [11] that narrow operators have a vector lattice nature. A number of results concerning linear narrow operators in ordered spaces were obtained in $[4,11-13]$. The state-of-the-art theory of narrow operators is presented in [14-17].

In these notes, the concept of the narrow operator is extended to the setting of bilinear operators. It is worth mentioning that different classes of bilinear operators on vector lattices were studied by different authors (see the survey article [18]).

In the first section of the article, the necessary information on vector lattices and bilinear regular operators is stated. In the next section, the notion of a bilinear narrow operator from the Cartesian product of Köthe-Banach spaces to a normed space is introduced and studied. It is proved that, for some conditions on Köthe-Banach spaces $E$ and $F$, the classes of function weakly narrow operators and narrow operators from $E \times F$ to a Banach space are coincident. The $C$-compact bilinear operators are investigated in the third section. It is shown that the $C$-compactness of a bilinear operator implies its narrowness. In the last part of the article, it is proved that a regular bilinear operator $T$ is narrow if and only if its modulus $|T|$ is. 
Author Contributions: Conceptualization, M.P.; Investigation, N.D. and R.K. All authors have read and agreed to the published version of the manuscript.

Funding: The research was funded by the Ministry of Science and Education of the Russian Federation (the number of agreement is 075-02-2021-1552).

Institutional Review Board Statement: Not applicable.

Informed Consent Statement: Not applicable.

Data Availability Statement: Not applicable.

Conflicts of Interest: The authors declare that there are no conflict of interest regarding the publication of this paper.

\section{References}

1. Popov, M.; Randrianantoanina, B. Narrow Operators on Function Spaces and Vector Lattices; De Gruyter Studies in Mathematics 45; De Gruyter: Berlin, Germany, 2013.

2. Abramovich, Y.A.; Aliprantis, C.D. An Invitation to Operator Theory; AMS: Providence, RI, USA, 2002.

3. Aliprantis, C.D.; Burkinshaw, O. Positive Operators; Springer: Dordrecht, The Netherlands, 2006.

4. Popov, M.; Sobchuk, O. On the "function" and "lattice" definition of a natrrow operator. Positivity 2018, 22, 59-62. [CrossRef]

5. Abasov, N. Completely additive and C-compact operators in lattice-normed spaces. Ann. Funct. Anal. 2020, 11, 914-928. [CrossRef]

6. Pliev, M. On C-compact orthogonally additive operators. J. Math. Anal. Appl. 2021, 494, 124594. [CrossRef]

7. Pliev, M.A.; Polat, F.; Weber, M.R. Narrow and C-compact orthogonally additive operators in lattice-normed spaces. Results Math. 2019, 74, 157. [CrossRef]

8. Kusraev, A.; Tabuev, S. On disjointness preserving bilinear operators. Vladikavkaz Math. J. 2004, 6, 58-70.

9. Lindenstrauss, J.; Tzafriri, L. Classical Banach Spaces. Vol. 2, Function Spaces; Springer: Berlin/Heidelberg, Germany; New York, NY, USA, 1979.

10. Plichko, A.; Popov, M. Symmetric function spaces on atomless probability spaces. Diss. Math. (Rozpr. Mat.) 1990, $306,1-85$.

11. Maslyuchenko, O.; Mykhaylyuk, V.; Popov, M. A lattice approach to narrow operators. Positivity 2009, 13, 459-495. [CrossRef]

12. Huang, J.; Pliev, M.; Sukochev, F. $l_{2}$ strictly singular operators on the predual of a hyperfinite von Neumann algebra. Proc. Amer. Math. Soc. 2021. [CrossRef]

13. Maslyuchenko, O.; Popov, M. On sums of strictly narrow operators acting from a Riesz space to a Banach space. J. Funct. Spaces 2019, 2019, 8569409. [CrossRef]

14. Abasov, N. On the sum of narrow orthogonally additive operators. Russ. Math. 2020, 64, 1-6. [CrossRef]

15. Fotiy, O.; Gumenchuk, A.; Krasikova, I.; Popov, M. On sums of narrow and compact operators. Positivity 2020, $24,69-80$. [CrossRef]

16. Pliev, M.; Popov, M. Narrow orthogonally additive operators. Positivity 2014, 18, 641-667. [CrossRef]

17. Pliev, M.; Sukochev, F. The Kalton and Rosenthal type decomposition of operators in Köthe-Bochner spaces. J. Math. Anal. Appl. 2021, 500, 125142. [CrossRef]

18. Bu, Q.; Buskes, G.; Kusraev, A.G. Bilinear maps on products of vector lattices: A survey. In Positivity, Trends in Mathematics; Birkhauser: Basel, Switzerland 2007; pp. 97-127. 